\title{
Features of gas excretion in wastewater treatment processes by the method of electroflotation
}

\author{
Evgeny Alekseev ${ }^{1, *}$ and Nadezhda Stashevskaya ${ }^{2}$ \\ ${ }^{1}$ Moscow State University of Civil Engineering, Yaroslavskoe shosse, 26, Moscow, 129337, Russia \\ ${ }^{2}$ Peoples' Friendship University of Russia (RUDN University), Miklukho-Maklaya street, 6, \\ Moscow, 117198, Russia
}

\begin{abstract}
The method of wastewater treatment by electroflotation is based on the electrochemical process of obtaining a gas dispersion. Features of the chemical composition of wastewater affect the electrochemical processes of water decomposition and the excretion of electrolysis gases. The aim of the research was to study the regularities of the separation of electrolysis gases from the ratio of the areas of polar electrodes and the value of the active reaction of the treated water $(\mathrm{pH})$. It is established that the optimum value of the ratio of the electrode areas $\left(f_{a}: f_{c}\right)$ close to 1 . The value of the current density at the electrodes is recommended to take in $150-200 \mathrm{~A} / \mathrm{m}^{2}$. An increase in the current density leads to heating of the liquid and an over-expenditure of electricity. The greatest influence of the $\mathrm{pH}$ of wastewater on the process of gas excretion is noted in the acidic medium. The gas yield is independent of the $\mathrm{pH}$ value in neutral and alkaline media. The gas yield remains practically unchanged with a current density of more than $150 \mathrm{~A} / \mathrm{m}^{2}$ over the entire range of $\mathrm{pH}$ changes from 2 to 12 .
\end{abstract}

\section{Introduction}

Electrochemical methods of wastewater treatment have a number of advantages and have become widespread in various industries [1-5]. Simple equipment is used for industrial implementation of electroflotation. During the operation of electroflotators, it is often not necessary to add additional reagents, which reduces the amount of forming sludges and precipitations that are subject to liquidation. A prerequisite for the use of electroflotators is the availability of electricity at any industrial facility. The prospects of its use have been established as a result of researches of the effectiveness of this method of wastewater treatment $[6,7]$. This is especially important for remote areas, the issues of environmental protection in which the use of simple and reliable methods of wastewater treatment is required. Many structural elements and technological parameters are taken without sufficient justification when designing and setting up plants, despite the simplicity of the

\footnotetext{
* Corresponding author: alekseeve@ @mgsu.ru
} 
electroflotation method for wastewater treatment. The basis of electroflotation is the process of electrochemical reception of the gas dispersion in the treated water [8].

The aim of the research was to study the dependency of the quantity of electrolysis gases obtained from such a constructive index as the ratio of the areas of polar electrodes, and also to study the effect of the value of active reaction of the treated water $(\mathrm{pH})$ on the process of gas excretion.

\section{Materials and methods of research}

Due to the fact that the aim of the research was to study only the stage of gas excretion, not the entire flotation process, experiments were carried out on an installation simulating the work of the electrode system of the flotator. Its structure made it possible to modify the active surfaces of the electrodes and distance between them. The installation included a horizontal gas-measuring device, which is a glass tube with a liquid plug. The value of the gas excretion was measured from the velocity of movement of the liquid plug. The advantage of this device is that, unlike other displacement systems, it has almost no effect on the pressure of the excreted gases. The electrode system was powered by a constant current source equipped with a potentiometer and a rheostat for changing the voltage and current, the values of which were recorded by electrical measuring instruments.

\subsection{Study of the influence of the ratios of the areas of polar electrodes}

When electric current passes through electrodes in electrolyte solutions (mineralized wastewater), the reactions occurring on their surfaces depend on the current density [9]. By definition, the current density is equal to the ratio of the current strength to the area of the active surface of the electrode:

$$
i_{a}=\frac{I}{f_{a}} \quad \text { and } \quad i_{c}=\frac{I}{f_{c}},
$$

where $i_{a}$ and $i_{c}$ - the current density at the anode and cathode, respectively, $\mathrm{A} / \mathrm{m}^{2}$; $f_{a}$ and $f_{c}$ - the active surface of the anode and the cathode, respectively, $\mathrm{m}^{2}$; $I$ - the value of the current, A.

The value of the current density, like any ratio, can take equal values for the most varied values of the current strength and the surface of the electrodes. Therefore, in the experiments, several values of the current were chosen for which we found the values of the gas yield, corresponding to different ratios of the densities of the anode and cathode currents. Under the condition of constant current, the ratio of the surface areas of the electrodes and the ratio of the current densities are inversely proportional. When the value of the current $I$ is constant, the following relation holds:

$$
\frac{f_{a}}{f_{c}}=\frac{i_{c}}{i_{a}}
$$

Under the conditions of the experiment, the change in the ratio of the areas was achieved by the different depth of immersion of the electrodes. The changes in the ratio $f_{a}: f_{c}$ was studied in the interval from 1:5 to 5:1. The current value varied from 0.1 to $1.0 \mathrm{~A}$. This corresponds to a change in the current density at the electrodes from 25 to $250 \mathrm{~A} / \mathrm{m}^{2}$.

Stainless steel plates for the cathode and electrical carbon plates for the anode were used as electrodes. The electrodes were not destroyed during operation. 


\subsection{Study of the influence of the active reaction of the medium $(\mathrm{pH})$}

The $\mathrm{pH}$ value in different wastewater varies within a wide range. Theoretically, the active reaction of the medium changes the electrode potentials, which should lead to the significant changes in the composition and the amount of excreted electrolysis gases [10].

The test solutions were prepared on the basis of the washing water of the textile factory after the dyeing process, the lavsan-viscose fabrics containing a set of salts into which a certain amount of sulfuric acid or sodium hydroxide was added to obtain solutions with $\mathrm{pH}$ $=2,4,6,8,10$ and 12 . Then the solution with the highest value of the electric conductivity value was chosen, over which the remaining solutions were leveled by adding the necessary amount of sodium sulfate. The decrease in the influence of side-effects on gas excretion was achieved in this way.

The study was carried out in the model of the electrode system of the electroflotator. Gas yield measurements were performed at the current values 0.3, 0.6, 0.9, 1.2 and $1.5 \mathrm{~A}$. This corresponds to the current densities at the cathode $30,60,90,120$ and $150 \mathrm{~A} / \mathrm{m}^{2}$.

\section{Results and discussions}

The results of experimental studies of the effect of the ratio of the areas of polar electrodes are presented in Fig. 1.

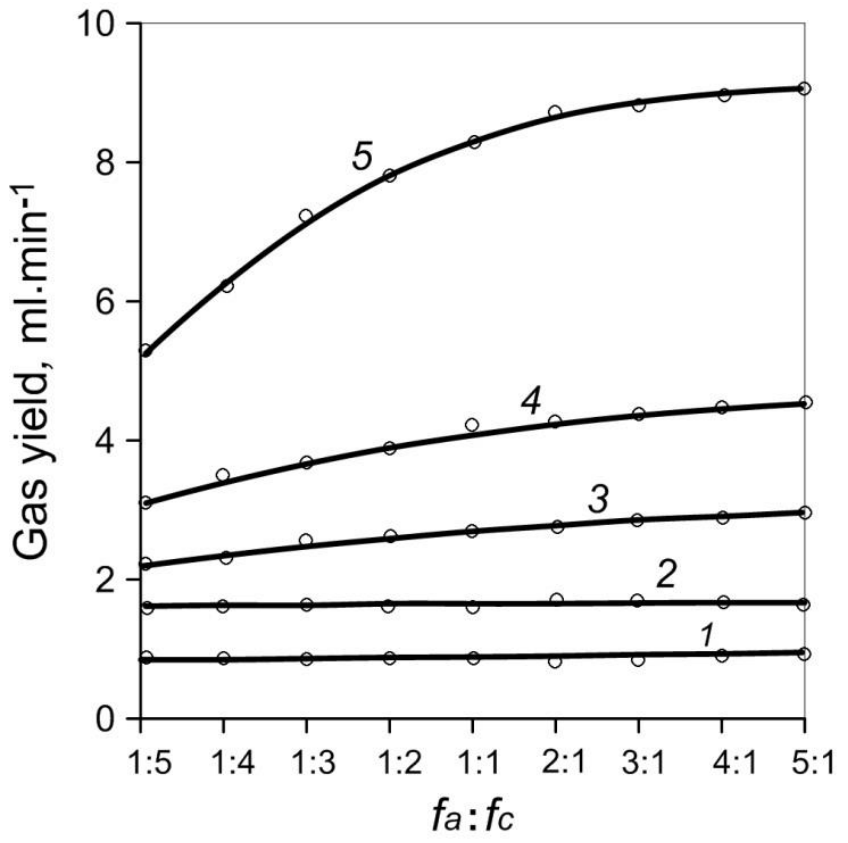

Fig. 1. Dependency of the gas yield on the ratio of the electrode areas and the current value: $1-0.1$ $\mathrm{A} ; 2-0.2 \mathrm{~A} ; 3-0.35 \mathrm{~A} ; 4-0.5 \mathrm{~A} ; 5-1.0 \mathrm{~A}$

In the range of low current density values from 25 to $50 \mathrm{~A} / \mathrm{m}^{2}$, corresponding to the current value of 0.1 to $0.2 \mathrm{~A}$, the ratio of electrode areas $\left(f_{a}: f_{c}\right)$ does not affect the gas yield.

A noticeable effect of the ratio of the electrodes areas to the gas yield occurs in the current density range of 85 to $150 \mathrm{~A} / \mathrm{m}^{2}$. With an increase in the current density of the cathode up to $250 \mathrm{~A} / \mathrm{m}^{2}$, the yield of gases exceeds the calculated value by almost two 
times. This is due to the fact that during the process of electrolysis at high current densities the intense heating of the liquid takes place at the electrodes up to the formation of water vapor, which was observed in the carried out experiments. When thermostating the electrode system model, the yield of electrolysis gases increases slightly.

An analysis of the obtained dependencies shows that the main part in the composition of electrolysis gases falls on hydrogen. The greatest gas yield is observed with the ratio of the areas of the electrodes corresponding to the value of the cathode current density of 150 $200 \mathrm{~A} / \mathrm{m}^{2}$. In addition, the results of this part of the studies demonstrate the low electrochemical scattering capacity of wastewater that used in the experiments. This should be taken into account when designing the electroflotators.

The results of the study of the influence of the active reaction of the medium $(\mathrm{pH})$ are shown in Fig. 2.

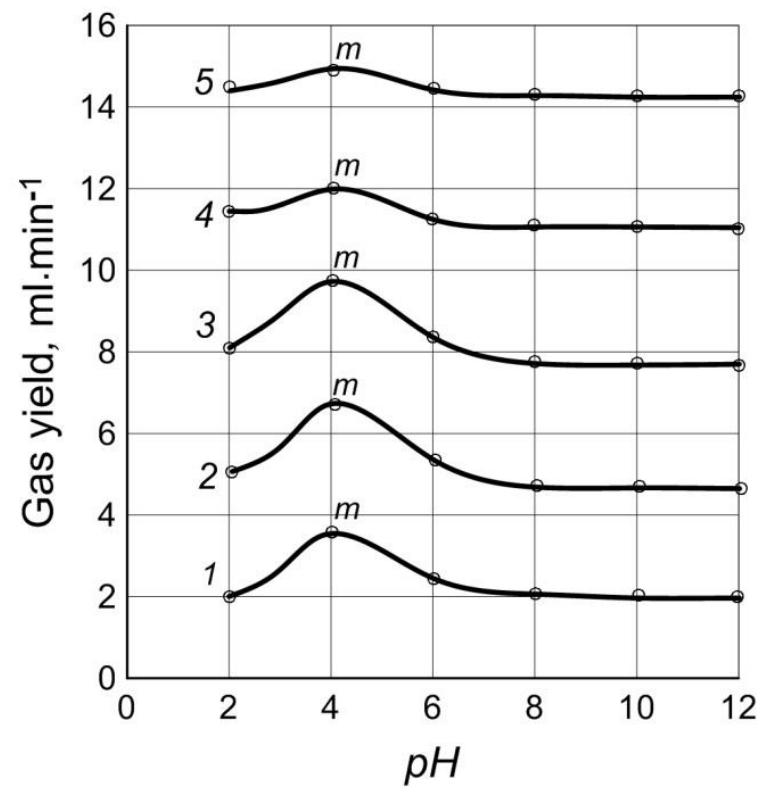

Fig. 2. Effect of $\mathrm{pH}$ of wastewater on the yield of electrolysis gases at current density: $1-30 \mathrm{~A} / \mathrm{m}^{2}$; $2-60 \mathrm{~A} / \mathrm{m}^{2} ; 3-90 \mathrm{~A} / \mathrm{m}^{2} ; 4-120 \mathrm{~A} / \mathrm{m}^{2} ; 5-150 \mathrm{~A} / \mathrm{m}^{2}$

In neutral and alkaline $\mathrm{pH}$ ranges, the gas yield is practically independent of the active reaction of the medium. Only an increase in gas excretion with increasing current is noted. In the range of $\mathrm{pH}$ values from 3 to 5 , the gas yield with the largest point "m" at $\mathrm{pH}=4$ increases. This dependency can be traced in the whole series of experiments. Thus, the ordinate of the point " $\mathrm{m}$ ", at the current value of $0.3 \mathrm{~A}$, is twice as large as the gas excretion value at other $\mathrm{pH}$ values. With increasing current density up to $150 \mathrm{~A} / \mathrm{m}^{2}$, a gradual smoothing of the maximum occurs.

The appearance of the maximum is obviously due to the presence of the chloride-ion in the studied solutions, the overvoltage of which assumes the minimum value in this electrochemical system at a $\mathrm{pH}$ equal to 4 . This assumption is confirmed by the analysis of electrolysis gases: a significant content (up to 10\%) of gaseous chlorine is noted at $\mathrm{pH}$ values close to 4 .

Despite the fact that, according to the theory of delayed discharge, hydrogen overvoltage should be a function of $\mathrm{pH}$, in this case it was not detected. Such a result is possible due to the presence of surfactant in solutions that exert a strong influence on the double electric layer. The smoothing of the maximum with increasing current density is 
caused by the change in the limiting stage in the process of gas excretion: the electrochemical mechanism of the process gradually turns into diffusion, since the mobility of the hydrogen ion is one of the highest.

\section{Conclusion}

When designing the electroflotation installations it is necessary to take into account that the ratio of the areas of polar electrodes does not significantly affect the amount of gas excretions, which is due to the low dissipative capacity of wastewater. Therefore, the optimal value of the ratio of electrode areas $\left(f_{a}: f_{c}\right)$ should be taken close to 1 . The value of the current density at the electrodes is recommended to take $150-200 \mathrm{~A} / \mathrm{m}^{2}$. When the current density is increased the liquid is heated, forms and releases water vapor, which unreasonably increases the power consumption.

In the range of low $\mathrm{pH}$ values of wastewater and low current densities on electrodes (30 $-90 \mathrm{~A} / \mathrm{m}^{2}$ ), the greatest amount of gas is released at the $\mathrm{pH}$ value of 4.0. The gas yield is independent of the $\mathrm{pH}$ value in neutral and alkaline media. The gas yield remains practically unchanged with the current density of more than $150 \mathrm{~A} / \mathrm{m}^{2}$ over the entire $\mathrm{pH}$ range of 2 to 12 .

\section{References}

1. G. Chen, Sep. Purif. Technol., 38, 11-41 (2004)

2. A.Y. Hosny, Sep. Technol., 6, 9-17 (1996)

3. G. Chen, X. Chen, P.L. Yue, J. Environ. Eng., 126(9), 858-863 (2000)

4. A. Khelifa, S. Aoudj, S. Moulay, M. De Petris-Wery, Chem. Eng. Process Intensif., 70, 110-116 (2013)

5. D.R. Ketkar, R. Mallikarjunan, S. Venkatachalam, Int. J. Miner. Proc., 31, 127-138 (1991)

6. M.M. De Santana, E.F. Zanoelo, C. Benincá, F.B. Freire, Process Safety and Environmental Protection, 116, 685-692 (2018)

7. G.Z. Kyzas, K.A. Matis, Journal of Molecular Liquids, 220, 657-664 (2016)

8. E.V. Alekseev, Ochistka stochnykh vod flotatsiei. Osnovy tekhnologii i primenenie: Монография [Sewage treatment by flotation. Fundamentals and application: Monograph] (ASV Publisher, Moscow, Russia, 2015)

9. D. Pletcher, R. Greff, R. Peat, L.M. Peter, J. Robinson, Instrumental Methods in Electrochemistry (Woodhead Publishing, Cambridge, UK, 2010)

10. C. Llerena, J.C.K. Ho, D.L. Piron, Chem. Eng. Commun., 155, 217-228 (1996) 